\title{
STRONG SOLUTIONS AND THE INITIAL DATA SPACE FOR SOME NON-UNIFORMLY PARABOLIC EQUATIONS
}

\author{
M. A. Skryabin \\ ITMO University, Saint Petersburg, Russia \\ mskryabin@my.ifmo.ru
}

PACS 02.30.Jr

DOI 10.17586/2220-8054-2015-6-1-146-153

This paper is devoted to strong solutions of the first and second initial-boundary problems for non-uniformly parabolic equations. These equations are used in mechanics, glaciology, rheology, image processing as well as for nanosystem modeling. The initial data space for these problems was explicitly described as OrliczSobolev spaces.

Keywords: non-uniformly parabolic equation, strong solution, initial data space, Orlicz-Sobolev space.

Received: 01 December 2014

Revised: 18 January 2015

\section{Introduction}

Let us consider the abstract Cauchy problem for the parabolic equation:

$$
\begin{gathered}
u^{\prime}(t)+A(u(t))=0, \quad t>0, \\
u(0)=u_{0} .
\end{gathered}
$$

The solution for this problem can be considered in different meanings, for example, a classical solution, strong solution, weak (or mild) solution, entropy solution, etc. This paper is devoted to a strong solution for this problem.

If $A$ is an unbounded linear operator in Hilbert space $H$, then it is proved that there does not exist a strong solution for all $u_{0} \in H$ (see $[2,11]$ ). It is proved in [13] that there exists a strong solution if and only if $u_{0} \in[D(A), H]_{1 / 2}$, where $[D(A), H]_{1 / 2}$ is an interpolation space (see $[9,15]$ ).

If $A$ is a nonlinear operator, the weak solutions of these problems is often investigated. We consider the case where the operator $A$ has the form:

$$
A(u)=-\operatorname{div}\left[\frac{\Phi^{\prime}(|\nabla u|)}{|\nabla u|} \nabla u\right]
$$

with the Dirichlet and Neumann boundary conditions for the first and second initialboundary problem respectively. Here, $\Phi: \mathbb{R} \rightarrow \mathbb{R}_{+}$is an $N$-function that satisfies $\Delta_{2}$ condition, therefore, it is natural to investigate these problems in Orlicz and Orlicz-Sobolev spaces. For these problems, we obtain the necessary and sufficient conditions for the existence and uniqueness of the strong solution.

Non-uniformly parabolic equations are used in mechanics, glaciology, rheology, image processing (e.g., see $[4,6,8,12]$ ) as well as for nanosystem modeling (see $[14,16]$ ). equations.

Now, we consider some examples of the function $\Phi(\xi)$ and corresponding nonlinear 
If $\Phi(\xi)=\xi^{p} / p, p>1$, then the nonlinear equation has the form:

$$
u_{t}-\Delta_{p} u=0,
$$

where $\Delta_{p} u=\operatorname{div}\left(|\nabla u|^{p-2} \nabla u\right)$ is the $p$-Laplace operator. The $p$-Laplacian type equations have many applications in fluid mechanics, glaciology, and rheology, etc. (see [4] and [8, Chapter 2]).

If $\Phi(\xi)=\xi \log (1+\xi)$, then we obtain the equation related to image processing. This special case has been investigated thoroughly in [17] as a model that developed Perona and Malik's concept in [12].

If $\Phi(\xi)=\xi \log \left(1+L_{k}(\xi)\right)$, where $L_{i}(\xi)=\log \left(1+L_{i-1}(\xi)\right)(i=1,2, \ldots, k)$ and $L_{0}(\xi)=\log (1+\xi)$, for $\xi \geq 0$, then we obtain the parabolic equation such that the corresponding elliptic problems are introduced in Prandtl-Eyring fluids and plastic materials with a logarithmic hardening law (see [6]).

\section{Functional Spaces}

In this section, we introduce functional spaces that are called Orlicz spaces and Orlicz-Sobolev spaces. The spaces $L_{\Phi}(\Omega)$, called Orlicz spaces, are studied in depth in the monograph by Krasnosel'skii and Rutickii [7] and also in the doctoral thesis by Luxemburg [10]. If the role played by $L_{p}(\Omega)$ in the definition of Sobolev space $W_{p}^{m}(\Omega)$ is assigned instead to an Orlicz space $L_{\Phi}(\Omega)$, the resulting space is denoted by $W^{m} L_{\Phi}(\Omega)$ and called an Orlicz-Sobolev space. Many properties of Sobolev spaces have been extended to Orlicz-Sobolev spaces, mainly by Donaldson and Trudinger [5].

Let $\phi$ be a real-valued function defined on $[0, \infty)$ and having the following properties:

(a) $\phi(0)=0, \phi(t)>0$ if $t>0, \lim _{t \rightarrow \infty} \phi(t)=\infty$;

(b) $\phi$ is nondecreasing;

(c) $\phi$ is right continuous.

Then, the real-valued function $\Phi(\xi)$ is defined on $[0, \infty)$ by:

$$
\Phi(\xi)=\int_{0}^{\xi} \phi(t) d t,
$$

which is called an $N$-function. It can be proved that any $N$-function is continuous, strictly increasing, and convex.

We set:

$$
\Phi^{*}(\eta)=\max _{\xi \geq 0}(\xi \eta-\Phi(\xi)) .
$$

If $\Phi$ is an $N$-function, then $\Phi^{*}$ is also an $N$-function. The function $\Phi^{*}$ is called the polar function.

An $N$-function $\Phi$ is said to satisfy $\Delta_{2}$-condition near infinity if there exists $\xi_{0}>0$ and a positive constant $M$ such that for every $\xi>\xi_{0}$ :

$$
\Phi(2 \xi) \leq M \Phi(\xi) .
$$

Let $\Omega$ be a bounded domain and $\Phi$ be an $N$-function. The Orlicz class is the set of all (equivalence classes modulo equality a.e. in $\Omega$ of) measurable functions $u(x)$ defined on $\Omega$ and satisfying:

$$
\int_{\Omega} \Phi(|u(x)|) d x<\infty .
$$

If $\Omega$ is a bounded domain, then the Orlicz class is a linear space (under pointwise addition and scalar multiplication) if and only if $\Phi$ satisfies $\Delta_{2}$-condition near infinity. Below, we 
consider only the case where $\Phi$ satisfies $\Delta_{2}$-condition near infinity. Note that the case where $\Phi$ does not satisfy $\Delta_{2}$-condition near infinity is more complicated.

If the Orlicz class is a linear space, then we can consider it as the Orlicz space $L_{\Phi}(\Omega)$ with the norm:

$$
\|u\|_{L_{\Phi}(\Omega)}=\inf \left\{k: \int_{\Omega} \Phi\left(\frac{|u(x)|}{k}\right) d x \leq 1\right\}
$$

(this norm is due to Luxemburg [10]). The space $L_{\Phi}(\Omega)$ is a Banach space.

We also introduce the space $W^{1} L_{\Phi}(\Omega)$ as completion of $C^{\infty}(\Omega)$ with respect to the norm:

$$
\|u\|_{W^{1} L_{\Phi}(\Omega)}=\|u\|_{L_{2}(\Omega)}+\|\mid \nabla u\|_{L_{\Phi}(\Omega)} .
$$

By $\stackrel{\circ}{W}^{1} L_{\Phi}(\Omega)$, we denote completion of $C_{0}^{\infty}(\Omega)$ with respect to the norm (5). Here, $C^{\infty}(\Omega)$ is the set of infinitely differentiable functions in $\Omega$ and $C_{0}^{\infty}(\Omega)$ is the set of infinitely differentiable functions in $\Omega$ with compact support. The space $W^{1} L_{\Phi}(\Omega)$ can be called an Orlicz-Sobolev space, but in our case, $W^{1} L_{\Phi}(\Omega) \subset L_{2}(\Omega)$. In the general case, it is not true (for example, see [1, Chapter VIII]).

\section{The First Initial-Boundary Problem}

Let $\Omega$ be a bounded domain in $\mathbb{R}^{n}(n \geq 2)$ with Lipschitz boundary $\partial \Omega$ and $T>0$. We denote $\Omega_{T}=\Omega \times(0, T)$ and $\Gamma=\partial \Omega \times(0, T)$. We consider the following parabolic equation

$$
u_{t}-\operatorname{div}\left[\frac{\Phi^{\prime}(|\nabla u|)}{|\nabla u|} \nabla u\right]=0 \quad \text { in } \Omega_{T},
$$

with the initial-boundary conditions

$$
\begin{aligned}
\left.u\right|_{\Gamma}=0 & \text { on } \Gamma, \\
u(x, 0)=u_{0}(x) & \text { in } \Omega .
\end{aligned}
$$

here $\Phi: \mathbb{R} \rightarrow \mathbb{R}_{+}$is an $N$-function, $\Phi^{\prime}: \mathbb{R} \rightarrow \mathbb{R}$ is the derivative of the function $\Phi(\xi)$ with respect to $\xi$, and $\nabla u$ is the gradient of the function $u(x, t)$ with respect to the spatial variables $x$. Problem (6)-(8) is called the first initial-boundary problem for a nonlinear equation.

Our main assumption is that $\Phi(\xi)$ and $\Phi^{*}(\xi)$ satisfy the $\Delta_{2}$-condition. We study the existence and uniqueness of a strong solution for problem (6)-(8). We assume that $u_{0} \in L_{2}(\Omega)$.

Definition 1. The function $u(x, t)$ is called a strong solution of problem (6)-(8) if the following conditions are met:

(1) $u \in L_{2}\left(\Omega_{T}\right) \cap L_{1}\left(0, T ; W_{1}^{1}(\Omega)\right)$,

$$
\int_{0}^{T} \int_{\Omega} \Phi^{\prime}(|\nabla u|)|\nabla u| d x d t<+\infty
$$

and $\left.u\right|_{\Gamma}=0$

(2) $u_{t} \in L_{2}\left(\Omega_{T}\right)$

(3) for every $v \in C^{1}\left(\bar{\Omega}_{T}\right)$ such that $\left.v\right|_{t=T}=0$ and $\left.v\right|_{\Gamma}=0$, we have:

$$
\int_{0}^{T} \int_{\Omega}\left[\frac{\Phi^{\prime}(|\nabla u|)}{|\nabla u|} \nabla u \cdot \nabla v-u v_{t}\right] d x d t=\int_{\Omega} u_{0}(x) v(x, 0) d x .
$$


Remark 1. If $u(x, t)$ is a strong solution of problem (6)-(8), then:

$$
\operatorname{div}\left[\frac{\Phi^{\prime}(|\nabla u|)}{|\nabla u|} \nabla u\right] \in L_{2}\left(\Omega_{T}\right)
$$

as well and the function $u(x, t)$ satisfies equation (6) a.e. in $\Omega_{T}$ and the initial condition (8) (in the sense of trace).

In [3], the uniqueness and existence of the weak solution were proven. In [18], the uniqueness and existence of the entropy solution were proven. These results were obtained if the function $\Phi$ satisfies weaker conditions than $\Delta_{2}$-condition.

Now we formulate the result concerning the strong solution.

Theorem 1. Let $\Phi(\xi)$ and $\Phi^{*}(\xi)$ satisfy the $\Delta_{2}$-condition near infinity. Then problem (6)(8) has a strong solution if and only if

$$
u_{0} \in \stackrel{\circ}{W}^{1} L_{\Phi}(\Omega) .
$$

The strong solution of problem (6)-(8) is unique.

Proof.

1. It is easy to show that the solution of problem (6)-(8) is unique (because the function $\Phi(\xi)$ is convex).

2. To prove an existence of a strong solution, we use the Faedo-Galerkin method. Let the system of functions $\left\{e_{k}\right\}_{k=1}^{\infty}, e_{k} \in C^{1}(\bar{\Omega}),\left.e_{k}\right|_{\Gamma}=0$ be linearly independent and full in $\stackrel{\circ}{W}^{1} L_{\Phi}(\Omega)$ (and in $L_{2}(\Omega)$ respectively).

We set $w_{N}(x, t)=\sum_{k=1}^{N} c_{N k}(t) e_{k}(x)(N=1,2, \ldots)$. The functions $w_{N}(x, t)$ are called the Faedo-Galerkin approximations and the coefficients $c_{N k}(t)$ can be found from the corresponding relations.

We obtain the following estimate for $w_{N}(x, t)$ :

$$
\begin{aligned}
& \int_{\Omega}\left|w_{N}(x, \tau)\right|^{2} d x \leq\left\|u_{0}\right\|_{L_{2}(\Omega)}^{2} \quad(\tau \in(0, T)), \\
& \int_{0}^{\tau} \int_{\Omega} \Phi^{\prime}\left(\left|\nabla w_{N}\right|\right)\left|\nabla w_{N}\right| d x d t \leq \frac{1}{2}\left\|u_{0}\right\|_{L_{2}(\Omega)}^{2} .
\end{aligned}
$$

Using (11), we also have:

$$
\left\|w_{N}\right\|_{L_{2}\left(\Omega_{T}\right)}^{2} \leq T\left\|u_{0}\right\|_{L_{2}(\Omega)}^{2} .
$$

From (12), (13) it follows that we can choose a subsequence (we also denote it by $w_{N}$ for simplicity) such that:

$$
w_{N} \rightarrow u \quad \text { weakly in } L_{2}\left(0, T ; \stackrel{\circ}{W}^{1} L_{\Phi}(\Omega)\right) .
$$

Moreover:

$$
\begin{gathered}
\|u\|_{L_{2}\left(\Omega_{T}\right)}^{2} \leq T\left\|u_{0}\right\|_{L_{2}(\Omega)}^{2}, \\
\int_{0}^{\tau} \int_{\Omega} \Phi^{\prime}(|\nabla u|)|\nabla u| d x d t \leq \frac{1}{2}\left\|u_{0}\right\|_{L_{2}(\Omega)}^{2} .
\end{gathered}
$$

We also have the following estimate for $\left\|w_{N t}\right\|_{L_{2}\left(\Omega_{T}\right)}$ :

$$
\left\|w_{N t}\right\|_{L_{2}\left(\Omega_{T}\right)}^{2} \leq \int_{\Omega} \Phi\left(\nabla w_{N}(x, 0)\right) d x .
$$


We have that $w_{N}(x, 0) \rightarrow u_{0}(x)$ as $N \rightarrow \infty$ weakly in $L_{2}(\Omega)$. Therefore, $\nabla w_{N}(x, 0) \rightarrow$ $\nabla u_{0}(x)$ as $N \rightarrow \infty$ weakly in $L_{2}(\Omega)$, i.e.:

$$
\int_{\Omega} \nabla w_{N}(x, 0) \eta(x) d x \rightarrow \int_{\Omega} \nabla u_{0}(x) \eta(x) d x,
$$

as $N \rightarrow \infty$ for an arbitrary function $\eta \in C(\bar{\Omega})$. Since the functions $\Phi(\xi)$ and $\Phi^{*}(\xi)$ satisfy the $\Delta_{2}$-condition, we obtain that $w_{N}(x, 0) \rightarrow u_{0}(x)$ as $N \rightarrow \infty$ weakly in $\stackrel{\circ}{W}^{1} L_{\Phi}(\Omega)$. Thus:

$$
\int_{\Omega} \Phi\left(\nabla w_{N}(x, 0)\right) d x \leq C
$$

where $C$ does not depend on $N$, and:

$$
\left\|w_{N t}\right\|_{L_{2}\left(\Omega_{T}\right)}^{2} \leq C
$$

Therefore, we can choose a subsequence (we also denote it by $w_{N}$ for simplicity) such that:

$$
w_{N t} \rightarrow u_{t} \text { weakly in } L_{2}\left(\Omega_{T}\right) .
$$

3. Now we need to verify that $u(x, t)$ is a solution for problem (6)-(8). This can be done by using the standard technique for the Faedo-Galerkin method.

4. We need to prove that if there exists a strong solution $u(x, t)$ of problem $(6)-(8)$, then the function $u_{0}(x)$ satisfies condition (10). It follows from these estimates:

$$
\begin{gathered}
\left\|u_{0}\right\|_{L_{2}(\Omega)}^{2} \leq C\left(\|u\|_{L_{2}\left(\Omega_{T}\right)}^{2}+\int_{0}^{T} \int_{\Omega} \Phi^{\prime}(|\nabla u|)|\nabla u| d x d t\right), \\
\int_{\Omega} \Phi\left(\nabla u_{0}(x)\right) d x \leq C\left(\left\|u_{t}\right\|_{L_{2}\left(\Omega_{T}\right)}^{2}+\|u\|_{L_{2}\left(\Omega_{T}\right)}^{2}+\left\|\operatorname{div}\left[\frac{\Phi^{\prime}(|\nabla u|)}{|\nabla u|} \nabla u\right]\right\|_{L_{2}\left(\Omega_{T}\right)}^{2}\right),
\end{gathered}
$$

where the constant $C$ does not depend on $u(x, t)$.

Theorem 1 is thus proved.

\section{The Second Initial-Boundary Problem}

Let $\Omega$ be a bounded domain in $\mathbb{R}^{n}(n \geq 2)$ with Lipschitz boundary $\partial \Omega$ and $T>0$. We denote $\Omega_{T}=\Omega \times(0, T)$ and $\Gamma=\partial \Omega \times(0, T)$. We consider the following parabolic equation:

$$
u_{t}-\operatorname{div}\left[\frac{\Phi^{\prime}(|\nabla u|)}{|\nabla u|} \nabla u\right]=0 \quad \text { in } \Omega_{T}
$$

with the initial-boundary conditions:

$$
\begin{array}{r}
\left.\frac{\partial u}{\partial \mathbf{n}}\right|_{\Gamma}=0 \quad \text { on } \Gamma, \\
u(x, 0)=u_{0}(x) \quad \text { in } \Omega .
\end{array}
$$

Here, $\Phi: \mathbb{R} \rightarrow \mathbb{R}_{+}$is an $N$-function, $\Phi^{\prime}: \mathbb{R} \rightarrow \mathbb{R}$ is the derivative of the function $\Phi(\xi)$ with respect to $\xi$, and $\nabla u$ is the gradient of the function $u(x, t)$ with respect to the spatial variables $x, \mathbf{n}$ is the unit normal vector of $\Gamma$. Problem $(16)-(18)$ is called the second initial-boundary problem for nonlinear equation.

Our main assumption is that $\Phi(\xi)$ and $\Phi^{*}(\xi)$ satisfy the $\Delta_{2}$-condition near infinity. We study the existence and uniqueness of a strong solution for problem (16)-(18). We assume that $u_{0} \in L_{2}(\Omega)$. 
Definition 2. The function $u(x, t)$ is called a strong solution of problem (16)-(18) if the following conditions are met:

(1) $u \in L_{2}\left(\Omega_{T}\right) \cap L_{1}\left(0, T ; W_{1}^{1}(\Omega)\right)$,

$$
\int_{0}^{T} \int_{\Omega} \Phi^{\prime}(|\nabla u|)|\nabla u| d x d t<+\infty
$$

(2) $u_{t} \in L_{2}\left(\Omega_{T}\right)$

(3) for every $v \in C^{1}\left(\bar{\Omega}_{T}\right)$ such that $\left.v\right|_{t=T}=0$, we have:

$$
\int_{0}^{T} \int_{\Omega}\left[\frac{\Phi^{\prime}(|\nabla u|)}{|\nabla u|} \nabla u \cdot \nabla v-u v_{t}\right] d x d t=\int_{\Omega} u_{0}(x) v(x, 0) d x .
$$

Remark 2. If $u(x, t)$ is a strong solution of problem (16)-(18), then:

$$
\operatorname{div}\left[\frac{\Phi^{\prime}(|\nabla u|)}{|\nabla u|} \nabla u\right] \in L_{2}\left(\Omega_{T}\right)
$$

as well and the function $u(x, t)$ satisfies equation (16) a.e. in $\Omega_{T}$ and the initial condition (18) (in the sense of trace).

In [17], the uniqueness and existence of the weak solution were proven if the function $\Phi(\xi)=\xi \log (1+\xi)$. This problem is related to image analysis.

Now, we formulate the results concerning the strong solution.

Theorem 2. Let $\Phi(\xi)$ and $\Phi^{*}(\xi)$ satisfy the $\Delta_{2}$-condition. Then problem (16)-(18) has a strong solution if and only if:

$$
u_{0} \in W^{1} L_{\Phi}(\Omega) .
$$

The strong solution of problem (16)-(18) is unique.

Proof.

1. It is easy to show that the solution of problem (16)-(18) is unique (because the function $\Phi(\xi)$ is convex).

2. To prove an existence of a strong solution, we use the Faedo-Galerkin method. Let the system of functions $\left\{e_{k}\right\}_{k=1}^{\infty}, e_{k} \in C^{1}(\bar{\Omega}),\left.e_{k}\right|_{\Gamma}=0$ be linearly independent and full in $W^{1} L_{\Phi}(\Omega)$ (and in $L_{2}(\Omega)$ respectively).

We set $w_{N}(x, t)=\sum_{k=1}^{N} c_{N k}(t) e_{k}(x)(N=1,2, \ldots)$. The functions $w_{N}(x, t)$ are called the Faedo-Galerkin approximations and the coefficients $c_{N k}(t)$ can be found from the corresponding relations.

We obtain the following estimate for $w_{N}(x, t)$ :

$$
\begin{aligned}
& \int_{\Omega}\left|w_{N}(x, \tau)\right|^{2} d x \leq\left\|u_{0}\right\|_{L_{2}(\Omega)}^{2} \quad(\tau \in(0, T)), \\
& \int_{0}^{\tau} \int_{\Omega} \Phi^{\prime}\left(\left|\nabla w_{N}\right|\right)\left|\nabla w_{N}\right| d x d t \leq \frac{1}{2}\left\|u_{0}\right\|_{L_{2}(\Omega)}^{2} .
\end{aligned}
$$

Using (21), we also have:

$$
\left\|w_{N}\right\|_{L_{2}\left(\Omega_{T}\right)}^{2} \leq T\left\|u_{0}\right\|_{L_{2}(\Omega)}^{2} .
$$

From (22) and (23) it follows that we can choose a subsequence (we also denote it by $w_{N}$ for simplicity) such that:

$$
w_{N} \rightarrow u \quad \text { weakly in } L_{2}\left(0, T ; \stackrel{\circ}{W}^{1} L_{\Phi}(\Omega)\right) .
$$


Moreover:

$$
\begin{gathered}
\|u\|_{L_{2}\left(\Omega_{T}\right)}^{2} \leq T\left\|u_{0}\right\|_{L_{2}(\Omega)}^{2} \\
\int_{0}^{\tau} \int_{\Omega} \Phi^{\prime}(|\nabla u|)|\nabla u| d x d t \leq \frac{1}{2}\left\|u_{0}\right\|_{L_{2}(\Omega)}^{2} .
\end{gathered}
$$

We also have the following estimate for $\left\|w_{N t}\right\|_{L_{2}\left(\Omega_{T}\right)}$ :

$$
\left\|w_{N t}\right\|_{L_{2}\left(\Omega_{T}\right)}^{2} \leq \int_{\Omega} \Phi\left(\nabla w_{N}(x, 0)\right) d x .
$$

We have that $w_{N}(x, 0) \rightarrow u_{0}(x)$ as $N \rightarrow \infty$ weakly in $L_{2}(\Omega)$. Therefore, $\nabla w_{N}(x, 0) \rightarrow$ $\nabla u_{0}(x)$ as $N \rightarrow \infty$ weakly in $L_{2}(\Omega)$, i.e.:

$$
\int_{\Omega} \nabla w_{N}(x, 0) \eta(x) d x \rightarrow \int_{\Omega} \nabla u_{0}(x) \eta(x) d x
$$

as $N \rightarrow \infty$ for an arbitrary function $\eta \in C(\bar{\Omega})$. Since the functions $\Phi(\xi)$ and $\Phi^{*}(\xi)$ satisfy the $\Delta_{2}$-condition, we obtain that $w_{N}(x, 0) \rightarrow u_{0}(x)$ as $N \rightarrow \infty$ weakly in $W^{1} L_{\Phi}(\Omega)$. Thus:

$$
\int_{\Omega} \Phi\left(\nabla w_{N}(x, 0)\right) d x \leq C
$$

where $C$ does not depend on $N$, and:

$$
\left\|w_{N t}\right\|_{L_{2}\left(\Omega_{T}\right)}^{2} \leq C
$$

Therefore, we can choose a subsequence (we also denote it by $w_{N}$ for simplicity) such that:

$$
w_{N t} \rightarrow u_{t} \text { weakly in } L_{2}\left(\Omega_{T}\right) .
$$

3. Now we need to verify that $u(x, t)$ is a solution of problem (16)-(18). This can be done using the standard technique for the Faedo-Galerkin method.

4. We need to prove that if there exists a strong solution $u(x, t)$ for problem (16)(18), then the function $u_{0}(x)$ satisfies condition (20). It follows from these estimates:

$$
\begin{gathered}
\left\|u_{0}\right\|_{L_{2}(\Omega)}^{2} \leq C\left(\|u\|_{L_{2}\left(\Omega_{T}\right)}^{2}+\int_{0}^{T} \int_{\Omega} \Phi^{\prime}(|\nabla u|)|\nabla u| d x d t\right) \\
\int_{\Omega} \Phi\left(\nabla u_{0}(x)\right) d x \leq C\left(\left\|u_{t}\right\|_{L_{2}\left(\Omega_{T}\right)}^{2}+\|u\|_{L_{2}\left(\Omega_{T}\right)}^{2}+\left\|\operatorname{div}\left[\frac{\Phi^{\prime}(|\nabla u|)}{|\nabla u|} \nabla u\right]\right\|_{L_{2}\left(\Omega_{T}\right)}^{2}\right),
\end{gathered}
$$

where the constant $C$ does not depend on $u(x, t)$.

Theorem 2 is thus proved.

\section{The Initial Data Space}

We define the initial data space as the space of the initial data such that there exists a strong solution only for them.

Theorem 3 (Main Result). If $\Phi(\xi)$ and $\Phi^{*}(\xi)$ satisfy the $\Delta_{2}$-condition, then:

(1) $W^{1} L_{\Phi}(\Omega)$ is the initial data space for the first initial-boundary problem (6)-(8);

(2) $W^{1} L_{\Phi}(\Omega)$ is the initial data space for the second initial-boundary problem (16)(18).

Proof. The proof clearly follows from Theorems 1 and 2. 


\section{Acknowledgements}

The author is grateful to Professor Shulin Zhou for his comments about this work. This work was supported by Beijing International Center for Mathematical Research.

\section{References}

[1] Adams R. A. Sobolev Spaces. Boston: Academic Press (1975).

[2] Ashyralyev A., Sobolevskii P.E. Well-Posedness of Parabolic Difference Equations. Basel, Boston, Berlin: Birkhäuser (1994).

[3] Cai Y., Zhou Sh. Existence and uniqueness of weak solutions for a non-uniformly parabolic equation. J. Functional Analysis, 257, P. 3021-3042 (2009).

[4] DiBenedetto E. Degenerate Parabolic Equations. Berlin: Springer-Verlag (1993).

[5] Donaldson T. K., Trudinger N. S. Orlicz-Sobolev spaces and imbedding theorems. J. Functional Analysis, 8, P. 52-75 (1971).

[6] Fuchs M., Mingione G. Full $C^{1, \alpha}$-regularity for free and contrained local minimizers of elliptic variational integrals with nearly linear growth. Manuscripta Math., 102, P. 227-250 (2000).

[7] Krasnosel'skii M. A., Rutickii Ya.B. Convex Functions and Orlicz Spaces. Noordhoff, Groningen, The Netherlands (1961).

[8] Lions J.-L. Some Methods of Solution of Nonlinear Boundary-Value Problems. Moscow: Mir (1972).

[9] Lions J.-L., Magenes E. Non-Homogeneous Boundary Value Problems and Applications. Berlin: Springer (1972).

[10] Luxemburg W. Banach function spaces. PhD Thesis, Technische Hogeschool te Delft, The Netherlands (1955).

[11] Pazy A. Semigroups of Linear Operators and Applications to Partial Differential Equations. New York: Springer-Verlag (1983).

[12] Perona P., Malik J. Scale-space and edge detection using anisotropic diffusion. IEEE Trans. Pattern Anal. Machine Intelligence, 12 (7), P. 629-639 (1990).

[13] Shamin R. V. Spaces of initial data for differential equations in a Hilbert space. Sb. Math., 194, P. 14111426 (2003).

[14] Shin K., Kang S. Doubly nonlinear parabolic equations involving p-Laplacian Operators via timediscretization method. Bull. Korean Math. Soc., 49 (6), P. 1179-1192 (2012).

[15] Tribel H. Interpolation Theory, Function Spaces, Differential Operators. Amsterdam: North Holland (1978).

[16] Umantsev A. Thermal effects of phase transformations: A review. Physica D, 235, P. 1-14 (2007).

[17] Wang L., Zhou Sh. Existence and uniqueness of weak solutions for a nonlinear parabolic equation related to image analysis. J. Partial Differential Equations, 19 (2), P. 97-112 (2006).

[18] Zhang Ch., Zhou Sh. Entropy solutions for a non-uniformly parabolic equation. Manuscripta Math., 131 (3-4), P. 335-354 (2010). 